\title{
Outstanding Improvement of the Advanced Lipoprotein Profile in Subjects with New-Onset Type 1 Diabetes Mellitus After Achieving Optimal Glycemic Control
}

\section{Esmeralda Castelblanco}

CIBER: Centro de Investigacion Biomedica en Red

Marta Hernández

Hospital Arnau de Vilanova

Emilio Ortega

Hospital Clinic de Barcelona

Nuria Amigó

Universitat Rovira i Virgili

Jordi Real

CIBER: Centro de Investigacion Biomedica en Red

Inka Miñambres

Hospital de la Santa Creu i Sant Pau

Carolina Lopez

Hospital Arnau de Vilanova

Albert Lecube

IRBLLEIDA: Institut de Recerca Biomedica de Lleida

Marcelino Bermúdez-López

IRBLLEIDA: Institut de Recerca Biomedica de Lleida

Núria Alonso

Hospital Universitari Germans Trias i Pujol

Josep Julve

Fundacio Institut de Recerca Hospital de la Santa Creu i Sant Pau: Hospital de la Santa Creu i Sant Pau Institut de Recerca

Didac Mauricio ( $\sim$ didacmauricio@gmail.com )

Hospital de la Santa Creu i Sant Pau Institut de Recerca https://orcid.org/0000-0002-2868-0250

Original investigation 
Keywords: Diabetic dyslipemia, Nuclear magnetic resonance (1H-NMR) spectroscopy, Cardiovascular risk, Lipoprotein remnants

Posted Date: May 11th, 2021

DOI: https://doi.org/10.21203/rs.3.rs-478979/v1

License: (c) (1) This work is licensed under a Creative Commons Attribution 4.0 International License. Read Full License

Version of Record: A version of this preprint was published at Diabetes Research and Clinical Practice on November 1st, 2021. See the published version at https://doi.org/10.1016/j.diabres.2021.109145. 


\section{Abstract}

Background: An altered lipoprotein profile is often hidden in normolipidemic subjects with uncontrolled type 1 diabetes mellitus (T1D). Conceivably, its amelioration after glycemic control may be overlooked in controlled T1D subjects. In this regard, we investigated the influence of glycemic optimization on lipoprotein subfraction parameters in normolipidemic new-onset T1D subjects.

Methods: Twelve subjects at onset of T1D ( 5 women and 7 men) were studied. Serum lipid and advanced lipoprotein profiles were determined by routine laboratory methods and $1 \mathrm{H}-\mathrm{NMR}$ spectroscopy shortly after diabetes diagnosis (baseline), and after achieving optimal glycemic control ( $\mathrm{HbA} 1 \mathrm{c}<7 \%$ ) at a followup of 11.3 to 25.6 weeks.

Results: Advanced lipoprotein analysis revealed a significant reduction from baseline in plasma concentrations of triglycerides (TG) and cholesterol (C), and in apolipoprotein (Apo)B-containing lipoproteins of treated subjects (VLDL-TG: $-21 \%$, LDL-TG: $-36 \%$, IDL-TG: $-32 \%$, LDL-TG: $-36 \%, P<0.05$; VLDL-C: $-46 \%$, IDL-C: -46\%, LDL-C: -16\%; P<0.05). Additionally, neither VLDL-TG and LDL-TG nor LDL-C of T1D subjects at follow-up differed from those of non-diabetic subjects. Decreased VLDL and LDL lipids were mainly attributed to concomitant reductions in the concentration of medium-sized VLDL $(-39 \%)$ and medium-sized LDL (34\%) and, to a lesser extent, to large-sized LDL (13\%), respectively. Notably, proatherogenic IDL characteristics and related surrogates of atherogenicity were resolved upon intensive glycemic control. However, neither the concentration of HDL-P, nor the relative proportion of HDL sizes was influenced by the glucose-lowering therapy.

Conclusions: In otherwise normolipidemic T1D subjects according to the conventional lipid profile, the achievement of optimal glycemic control after diabetes onset is associated with normalization of profound derangements in ApoB-containing lipoproteins (VLDL, IDL, and LDL). Whether higher cardiovascular risk in poorly controlled T1D is partially mediated by these proatherogenic lipid alterations should be elucidated by further studies.

\section{Background}

Type 1 diabetes mellitus (T1D) is associated with premature cardiovascular disease (CVD) [1]. Hyperglycemia is a hallmark of T1D and a major independent risk factor for CVD in this type of diabetes [2]. The contribution of other hidden additional risk factors further elevates CVD risk in a synergistic manner in T1D [2], and their management has been prioritized in recent updates of the American Diabetes Association (ADA) and the European Association (EASD) recommendations [3, 4]. In this regard, accumulating evidence suggests that glycemic control has been strongly associated with improved CVD outcomes in T1D $[2,5,6]$. Although the underlying mechanisms remain insufficiently understood, a significant part of the cardioprotective effect of intensive glucose-lowering is likely related to the favorable effects on lipoprotein metabolism $[7,8]$. 
An altered, proatherogenic lipoprotein profile is often obscured by apparently normal serum concentrations of total triglycerides (TG), total cholesterol, low-density lipoprotein and high-density lipoprotein cholesterol (LDL-C and HDL-C, respectively) in subjects with poorly controlled T1D [2, 9-11]. Additionally, normal LDL-C values may coexist with increased intermediate-density lipoproteins (IDL), and small and dense LDL (sdLDL) in T1D subjects $[12,13]$. Likewise, HDL-C values may be normal or even moderately elevated in T1D subjects $[11,14,15]$, but HDL in diabetes may display poorer antiatherogenic properties $[16,17]$, due at least in part to the cumulative associated glycoxidative modifications of $\mathrm{HDL}$ proteins [14].

Advanced lipoprotein analysis using nuclear magnetic resonance (1H-NMR) spectroscopy allows the screening and characterization of circulating lipoprotein classes $[11,18]$, and the identification of specific lipoprotein characteristics, such as size and composition, provided by this advanced analysis may be potentially useful in timely and accurately predicting CVD risk in subjects with T1D.

The use of advanced lipoprotein analysis to more accurately evaluate parameters of lipid metabolism beyond the classical clinical lipid profile may yield relevant information of how glycemic regulation is influencing lipid metabolism in T1D. Supporting this view, optimization of glycemic control with intensive therapy significantly decreases sdLDL in subjects with poorly controlled T1D with a mean disease duration of $1.7 \pm 3.5(0-16)$ years [15]. In a recent cross-sectional study that included different cohorts, lipoprotein analysis by $1 \mathrm{H}$-NMR spectroscopy revealed reduced serum concentrations of apolipoprotein (Apo)B-containing lipoproteins, both very-low-density lipoprotein (VLDL) and LDL particles (VLDL-P and LDL-P), and concomitant lower levels of VLDL-TG and non-HDL-C in subjects with T1D [18]; however, in the latter study, T1D participants were on intensified insulin therapy for $>1$ year and/or $\geq 10$ years, and thus the favorable impact, if any, of the improvement of glycemic control on their lipoprotein profile in the same individual could not be assessed with this cross-sectional design. Therefore, we tested the hypothesis that optimization of glycemic control would improve the extended serum lipoprotein profile of subjects with new-onset T1D by assessing the advanced lipoprotein profile before and after the optimization glycemic control.

\section{Methods}

\section{Participants}

This was a two-center before-and-after study performed at the University Hospital Arnau de Vilanova (Lleida) and the University Hospital Germans Trias i Pujol (Badalona) in northwestern Spain. The study

protocol was approved by the Ethics Committees of both participating centers (CEIC-1571 and PI-15-147, respectively), and all participants were informed and gave their consent to participate in the study.

T1D subjects included in the present study were part of a before-and-after study primarily designed to assess the metabolomic changes induced by hyperglycemia in new-onset T1D and by its intensive treatment. The inclusion criteria were as follows: adults ( $\geq 18$ years) with a new clinical diagnosis of T1D 
according to the ADA criteria who provided written informed consent. Importantly, serum sampling in new-onset T1D subjects was done after full recovery from ketosis/ketoacidosis (i.e., absence of ketonemia was defined as $<0.5 \mathrm{mmol} / \mathrm{L}$ ) to prevent further metabolic masking and, thus, assess the direct effect of previous exposure to severe hyperglycemia on the lipoprotein phenotype. Additionally, a pre-specified $\mathrm{HbA} 1 \mathrm{c}$ target of $<7 \%$ at follow-up was established; thus, subjects not fulfilling this criterion after a maximum time period of 26 weeks were excluded from the study. Consequently, a small subset of T1D subjects $(\mathrm{N}=12)$ was eventually included to analyze the impact of intensive glycemic control on

lipoprotein subclasses. According to this rationale, this study should be considered as an exploratory pilot sub-study. Exclusion criteria at onset were: presence of dehydration or ketosis, and previous history of dyslipidemia, hypertension, chronic kidney disease or any form of cardiovascular disease.

As a control group, we selected 12 non-diabetic subjects ( $\mathrm{HbA} 1 \mathrm{c}<5.7 \%$ and fasting glucose $<100 \mathrm{mg} / \mathrm{dL}$ ) matched for gender, age, and body mass index (BMI) to the T1D subjects after optimization of glycemic control. This control group was used as a reference group to compare the benefits of intensive therapy on the lipoprotein profile in this subset of T1D subjects (Additional file 1). Exclusion criteria for the control subjects were the same as those considered for the T1D subjects.

\section{Clinical and conventional biochemical determinations}

A thorough clinical assessment was performed, including the collection of relevant parameters including weight, waist, and BMI. Fasting blood samples were obtained for all biochemical measurements. Glucose and glycated hemoglobin (HbA1c) were determined using standard procedures. The glomerular filtration rate (GFR-CKD-EPI) was calculated according to Chronic Kidney Disease Epidemiology Collaboration (CKD-EPI) equation [19]. Serum samples were stored at $-80^{\circ} \mathrm{C}$ until analysis. Standard laboratory analysis included total cholesterol and triglycerides (TG), and LDL-C and HDL-C. ApoB and ApoA-I, and Lp(a) analyses were performed enzymatically using commercial kits (Roche Diagnostics SL, Sant Cugat del Vallès, Spain), and non-esterified fatty acids (NEFA) were determined using reagents from Wako Diagnostics (Wako Chemicals, Osaka, Japan) adapted for a COBAS c501 autoanalyzer (Roche Diagnostics SL, Sant Cugat del Vallès, Spain). Both were considered to further confirm favorable lipoprotein changes and insulin therapy in intensified T1D subjects.

\section{Advanced serum lipoprotein profile}

Advanced lipoprotein analysis was performed in serum samples using the Liposcale ${ }^{\circledR}$ test (Biosfer Teslab, Reus, Spain), a 2D diffusion-ordered 1H-NMR spectroscopy-based method, as previously described [20]. The advanced analysis included the determination of lipid (TG and cholesterol) concentrations, size of three different classes of lipoproteins (very-low-density lipoprotein (VLDL), LDL and $\mathrm{HDL}$ ) as well as the particle concentration of nine subclasses (large, medium and small VLDL, LDL and $\mathrm{HDL}$ ). The method also included the evaluation of IDL lipid concentrations. Briefly, 2D 1H-NMR spectra were recorded on a Bruker Avance III 600 spectrometer, operating at a proton frequency of 600 $\mathrm{MHz}$ at $310 \mathrm{~K}$ (Bruker BioSpin, Rheinstetten, Germany). The methyl signal was surface-fitted with nine functions associated with specific lipoprotein subclasses according to its NMR size. The particle number 
of each main lipoprotein fraction was calculated by dividing the lipid volume by the particle volume of a given class and weighted average VLDL, LDL and HDL particle sizes (in nm diameter units) were determined [21]. In relation to the atherogenic indexes, non-HDL-P, the sum of all ApoB-containing lipoprotein classes, the total-P/HDL-P ratio, and the LDL-P/HDL-P ratio were evaluated. In addition, the following were also determined: atherogenic particles (VLDL-P + LDL-P); the atherogenic particles/HDL-P ratio, atherogenic cholesterol (as VLDL-C + IDL-C and LDL-C); the atherogenic cholesterol/HDL-C ratio; and remnant cholesterol (VLDL-C + IDL-C).

\section{Statistical analysis}

Sample size ( $n=12$ per group) was determined by the availability of subjects with confirmed intensive optimization of glycemic control in a sub-cohort of T1D subjects selected from a previous study. The number of subjects included in our study was sufficient to assess the changes in NMR-assessed lipoprotein subclass profiles.

Data are presented as percentage for categorical variables, and as mean and standard deviation (SD) for continuous variables. Standardized differences (effect size) were computed to assess the magnitude of changes between clinical and lipoprotein parameters using the Cohen's $d$ function of effect-size package (version 0.4.1) using the free R statistical software version 3.6.0. (https://www.r-project.org/). A test significance of global changes was performed for each table (Clinical, laboratory, lipid content, etc.) using non-parametric Sign test. $P$ values of each specific sub variable were computed using post hoc t-test corrected for Benjamini \& Hochberg (false discovery rate). Differences between groups were analyzed using independent t-test between groups. A p-value $<0.05$ was considered as statistically significant at a family-wise level. Pearson correlation coefficients were used to assess the relationship between changes in NMR-assessed lipoprotein subclass profiles and changes in conventional clinical/biochemical variables in treated T1D subjects. A heat map was created to show the correlations of changes, and a forest plot was generated to show the relative changes in NMR-assessed lipoprotein subclass profiles. Calculations and plots were made using the above-mentioned version of the free $\mathrm{R}$ statistical software. Data of this study is available via open code at the following cloud domain: https://github.com/jrealgatius/LIPOS_DEBUTS.

\section{Results}

A total of 20 subjects with T1D who fulfilled the inclusion criteria were initially recruited after signing the informed consent. However, 8 subjects were excluded, 6 for not achieving the pre-defined target HbA1c at follow-up, and 2 who were lost to follow-up. For the remaining 12 subjects, all were assessed in the outpatient clinic within 7 days after the diagnosis of T1D.

\section{Clinical and conventional lipid profile analysis in subjects with T1D}

The clinical characteristics of the study T1D subjects are shown in Table 1. Optimization of glycemic control in subjects with T1D was shown by HbA1c levels of $6.2 \pm 0.5 \%$, being the highest $\mathrm{HbA} 1 \mathrm{c}$ value at 
follow-up 6.7\%. Body mass index was increased after intensified optimization of glycemic control. Conventional biochemical profiles did not differ among groups, except for plasma glucose and HbA1c. Lipid parameters determined in subjects with onset T1D were within established normal ranges, except for a female with a TG concentration of $239 \mathrm{mg} / \mathrm{dL}$ and 2 males with lower baseline HDL-C (32 and 35 $\mathrm{mg} / \mathrm{dL}$, respectively) (Additional file 2). Overall, the conventional lipid profile normalized after the intensive management of glycemic control except in one subject who had a mild increase in the concentration of TG $(163 \mathrm{mg} / \mathrm{dL})$ (Additional file 2). The only meaningful change in the conventional lipid profile produced by intensively optimized glycemic control was a significant decrease in the LDL-C (-16\%; $p=0.011$ ) (Table 1). ApoB was consistently lower at follow-up $(-21 \%, p=0.002)$ in T1D (Additional file 3). Despite this, total cholesterol was just marginally reduced in subjects after achieving optimal glycemic control ( $p=0.054)$ compared with baseline concentrations (Table 1). Additionally, serum concentrations of both TG and NEFA did not significantly differ between baseline and follow-up (Table1, Additional file 3).

Of note, $\mathrm{HbA} 1 \mathrm{c}$ values showed a close-to-significant trend to be increased $(p=0.056)$ in T1D subjects (6.2\%) compared with non-diabetic subjects (5.2\%), even with intensified optimization of glycemic control (Additional file 1).

\section{Advanced NMR analysis of the serum lipoprotein profile of subjects with T1D}

Advanced 1H-NMR analysis yielded important changes in the lipoprotein profile in T1D subjects after intensive glycemic control (Table 2). Indeed, the serum concentration of non-HDL-P, which is representative of total ApoB-containing lipoproteins, was significantly decreased $(-17 \%, p=0.005)$, as was the sum of atherogenic particles $(-16 \%, p=0.006)$ (Additional file 4).

Total serum levels of VLDL-P did not change after glycemic optimization. However, the concentration of medium VLDL-P (representing about $10 \%$ of the three VLDL subclasses) was significantly reduced after achieving optimal glycemic control $(-39.5 \%, p=0.033)$ (Table 3$)$. This finding was accompanied by a concomitant decrease in the serum levels of total VLDL-TG and VLDL-C $(-22 \%, p=0.021$ and $-23 \%$, $p=0.045$, respectively) and in the IDL-C $(-46 \%, p<0.001)$ and IDL-TG concentrations $(-30 \%, p<0.001)$ (Table 2). Of note, the ratio IDL-C/IDL-TG was $23 \%$ lower in T1D subjects after glycemic control optimization (Additional file 5). Taken together, total circulating atherogenic remnants, which may be inferred by the sum of both VLDL and IDL classes, were favorably influenced $(-35 \%, p=0.002)$ by optimization of glycemic control (Additional file 4).

Consistent with data from the conventional lipid profile (Table 1), serum concentrations of LDL-C were significantly lower $(-16 \%, p=0.011)$ at follow-up (Table 2$)$. In line with this, the serum concentration of atherogenic cholesterol was concomitantly lower $(-19 \%, p=0.002)$ (Additional file 4). Similar results were found for serum LDL-TG levels at follow-up compared to the baseline $(-36 \%, p<0.001)$ (Table 2$)$. At followup, favorable reductions in circulating LDL lipids were further accompanied by concomitant decreases in the total LDL-P $(-16 \%, p=0.007)$ which were mainly accounted for by a reduction in large- and mediumsized LDL-P subclasses concentrations $(-12.9 \%, p=0.036$ and $-34 \%, p=0.007$, respectively) (Table 3 ). 
Regarding HDL, serum levels of HDL-TG were significantly decreased in T1D subjects $(-18 \%, p=0.045)$ after reaching optimal glycemic control, but did not significantly influence serum concentrations of HDL-C (Table 2). In line with this, the HDL-TG-to-HDL-P ratio was concomitantly decreased after optimization $(-39 \%, p=0.003)$ whereas that of HDL-C-to-HDL-TG increased compared with baseline values (Additional file 5).

Improved serum lipoprotein ratios calculated at follow-up in T1D subjects were mainly attributed to favorable reductions in the non-HDL component (LDL-P-to-HDL-P ratio: 0.82-fold, $p=0.004$; atherogenic particles (VLDL-P + LDL-P)-to-HDL-P ratio: 0.81-fold, $p=0.004$; atherogenic cholesterol-to-HDL-C ratio: 0.77 -fold, $p=0.002$ ) (Additional file 4). Additionally, the ratio IDL-C-to-IDL-TG was significantly decreased in T1D subjects after intensive therapy $(-23 \%, p=0.001)$ (Additional file 5 ).

\section{Correlation between the changes produced after optimization of glycemic control on changes in serum lipid profile with anthropometric and clinical variables in subjects with T1D}

Favorable changes of most VLDL characteristics, including concentrations and composition of circulating VLDL subclasses, were positively associated with improved HbA1c values (Additional file 6). Though moderately, the IDL lipids and circulating remnants (non-HDL-P) were also associated with favorable changes in HbA1c. Interestingly, large-sized (buoyant) LDL-P was inversely associated with leukocyte counts, a surrogate of inflammation. No additional significant relationships among lipoprotein characteristics and clinical or biochemical variables were seen, except for those between the HDL-C-toHDL-TG ratio and liver gamma-glutamyl transferase.

\section{Forest plot}

This analysis showed changes in advanced lipoprotein characteristics during glycemic control in subjects with T1D (Additional file 7). Interestingly, the effect size of intensified glycemic control optimization was significantly larger in LDL and IDL than that observed in the VLDL.

\section{Discussion}

Current evaluation of lipid abnormalities often fails to uncover the full lipoprotein atherogenicity in normolipidemic subjects with T1D under conventional metabolic management [15, 22-27]. We herein show for the first time the potential of $1 \mathrm{H}-\mathrm{NMR}$ spectroscopy to unveil hidden favorable changes in the whole spectrum of circulating ApoB-containing lipoprotein classes and lipoprotein atherogenicity in new onset T1D subjects after the achievement of optimal glycemic control.

Our data is consistent with previous studies from our group [18, 27] and others [15, 23-25] showing favorable reductions in the serum levels of ApoB-containing lipoproteins, VLDL and LDL, and elevations in HDL in T1D subjects compared with matched non-diabetic subjects. However, a favorable, less proatherogenic change in serum IDL in T1D subjects after optimized glycemic control in T1D subjects may have been overlooked in the previous studies $[18,27]$. 
Serum concentrations of different classes of ApoB-containing lipoproteins were overall decreased in T1D subjects after optimal glycemic control (Additional file 1). Consistently, this finding was accompanied by a concomitant reduction of serum concentrations of $A p o B$ (Additional file 3 ), which is a surrogate of the total number of circulating non-HDL particles. In line with previous data [28], significant reductions in cholesterol transported by ApoB-containing lipoproteins (Table 2, Additional file 4) would reveal an improved atherogenicity of these lipoproteins. Additionally, our data also showed a significant delipidization in all the ApoB-containing lipoproteins. Increased TG content in these lipoprotein classes is a marker for several types of atherogenic lipoproteins [29], and elevations of TG-rich lipoproteins, including VLDL and IDL (VLDL remnants), promote atherogenesis independently of LDL [30]. TG reductions are accompanied by a favorable reduction of cardiovascular risk in T1D subjects after glycemic control optimization.

IDL are proatherogenic cholesterol-laden lipoproteins contributing to remnant cholesterol and may frequently accumulate in the serum of several dyslipidemic disorders associated with accelerated atherosclerosis [31]. Additionally, elevated IDL may coexist with apparently normal levels of serum LDL levels and is considered an independent risk factor for aortic atherosclerosis in subjects on hemodialysis [32]. As occurs in the current study subjects, higher serum levels of IDL-C increase the atherogenic risk in normolipidemic T2D subjects [33,34]. Importantly, glycemic optimization produced a favorable decrease in IDL-C and IDL-TG and thus atherogenic remnants, which in turn improved lipoprotein atherogenicity indexes in controlled T1D subjects. Because the lipoprotein profile of T1D subjects used in our previous study were analyzed in subjects with a reasonable diabetic control [11], IDL could plausibly be within the normal range defined by the non-diabetic subjects and thus not detected even in the case that serum IDL$C$ could have been eventually measured. Of note, these data highlight the importance of measuring serum IDL-C and IDL-TG to further characterize the atherogenic lipoprotein profile in subjects with T1D and thus their contribution to elevated CVD risk.

Some VLDL characteristics were inversely related to waist circumference in optimized T1D subjects. Intriguingly, these same VLDL parameters have been positively related to components of metabolic syndrome, i.e., BMI, waist circumference, inflammatory state, and fatty liver index in a cohort of wellcontrolled T1D subjects [27]. The reason for this discrepancy is unknown, but it could, at least partly, be due to differences in the study design. Indeed, our study provides data on dynamic changes in serum medium and large VLDL-P levels and its negative relationship with the magnitude of change in the waist circumference of our T1D subjects in response to glycemic control optimization, whereas the previous study shows a steady-state relationship between both parameters [27].

Hyperglycemia also profoundly affects HDL metabolism [35]. Indeed, glycated ApoA-I is a main determinant of the atheroprotective actions of HDL. Moreover, glycated ApoA-I also reduces the clearance of HDL from circulation, which could conceivably explain the elevated trend towards increased serum concentrations of HDL-C in T1D subjects even after glycemic control optimization compared with nondiabetic subjects (Additional file 1). On the other hand, TG enrichment of HDL, a hallmark of T1D at onset [35], was reversed with optimal glycemic control in our T1D subjects (Additional file 5). The latter could be 
attributed to increased hepatic lipase $(\mathrm{HL})$ activity, among other factors. HL hydrolyzes TG from IDL/LDL and also HDL [36]. Interestingly, its release is positively influenced by insulin [37-40], and hence it could possibly be induced in optimized T1D subjects in our study, and potentially underlie the decreased TG content in both IDL/LDL and, to a lesser extent, HDL.

The strengths of this study include the fact that it is focused on intensified optimization of glycemic control of T1D, and its longitudinal nature. T1D subjects were apparently sufficiently insulin treated, as revealed by well-resolved ketosis/ketoacidosis (showing normal serum levels of TG at baseline) and an absence of changes in the serum concentrations of NEFA (Additional file 3). Limitations include the relatively small sample size used, contributing to the pilot nature of the study. Only T1D participants reaching glycemic intensification criteria (with $\mathrm{HbA} 1 \mathrm{c}$ values below 6.5\%) were included, and thus the results may not be representative of the entire T1D population. The latter may limit the interpretation of the observations and should therefore be considered with caution. Thus, the favorable changes in ApoBcontaining lipoproteins, particularly the proatherogenic IDL, should be confirmed in future studies with a larger number of subjects.

\section{Conclusion}

The current work reinforces the value of determining the advanced lipoprotein profile for the study of lipid abnormalities in T1D. Optimal glycemic control in very poorly controlled T1D subjects favorably influences lipoprotein disturbances. Importantly, the net effect of hyperglycemia in new-onset T1D subjects was analyzed without the contribution of other metabolic confounders and the $1 \mathrm{H}-\mathrm{NMR}$ approach unveiled a relevant improvement in the atherogenic characteristics of lipoproteins, mainly involving ApoB-containing lipoproteins, which often remain undetected using conventional methodologies. Remarkably, the advanced lipoprotein analysis also unveiled favorable changes in the atherogenic IDL characteristics after glycemic optimization. Further studies are warranted to validate the atheroprotective implications of these conclusions.

\section{Abbreviations}

1H-NMR: nuclear magnetic resonance; CKD-EPI: Chronic Kidney Disease Epidemiology Collaboration; CVD: cardiovascular disease; IDL: intermediate-density lipoproteins; NEFA: non- esterified fatty acids; sdLDL: small and dense LDL; T1D: type 1 diabetes mellitus; TG: triglycerides; VLDL: very-low-density lipoprotein.

\section{Declarations}

\section{Ethics approval and consent to participate}

The study protocol was approved by the Ethics Committees of University Hospital Arnau de Vilanova (Lleida) (CEIC-1571) and the University Hospital Germans Trias i Pujol (Badalona) (PI-15-147), and all 
participants were informed and gave their consent to participate in the study.

\section{Consent for publication}

Not applicable.

\section{Availability of data and materials}

The dataset generated and/or analyzed during the current study ar available in the GitHub repository, https://github.com/jrealgatius/LIPOS_DEBUTS.

\section{Competing interests}

N. Amigó is stock owner of Biosfer Teslab and has a patent of the lipoprotein profiling described in the present manuscript. The rest of authors declare that they have not competing interests.

\section{Funding}

This work was funded by Ministerio de Sanidad y Consumo, Instituto de Salud Carlos III (Madrid, Spain) grant PI15/0625 (to D.M. and E.C.), PI17/01362 (to NA), and PI17/00232 (to JJ), FEDER "Una manera de hacer Europa”, and by Fundació La Marató de TV3 2016 (303/C/2016) (201602.30.31) (to N.A and J.J.). J.J. was recipient of a Miguel Servet Type 2 contract (CPII18/00004; ISCIII). CIBER de Diabetes y Enfermedades Metabólicas Asociadas (CIBERDEM) is a project of Instituto de Salud Carlos III. J.J. is member of Red de Investigación en "Enfermedades Metabólicas y Cáncer" (RED2018-102799-T), Ministerio de Economía y Competitividad (MINECO), Madrid, Spain. Institut de Recerca de l'Hospital de la Santa Creu i Sant Pau is accredited by the Generalitat de Catalunya as Centre de Recerca de Catalunya (CERCA).

\section{Authors' contributions}

EC and DM designed and conceptualized the study. EC, MH, and NAm contributed researched data; EC, $\mathrm{MH}, \mathrm{EO}, \mathrm{NA}, \mathrm{IM}, \mathrm{CL}, \mathrm{AL}, \mathrm{MB}-\mathrm{L}, \mathrm{NA}, \mathrm{JJ}$, and DM interpreted data; EO, NAm, and NA. contributed to the discussion and reviewed the manuscript; $\mathrm{EC}, \mathrm{JJ}$, and $\mathrm{DM}$ wrote the original draft of the manuscript; $\mathrm{EC}$, $\mathrm{MH}, \mathrm{EO}, \mathrm{NAm}, \mathrm{NA}, \mathrm{JJ}$, and DM reviewed/edited the manuscript; DM supervised the study; funding acquisition was provided by $\mathrm{NA}, \mathrm{JJ}$, and DM. All authors read and approved the manuscript.

\section{Acknowledgements}

We want to particularly acknowledge the participants, and the IGTP-HUGTP and IRBLleida (B.0000682) Biobanks integrated in the Spanish National Biobanks Network of ISCIII (PT17/0015/0045 and PT17/0015/0027, respectively).

\section{References}


1. Petrie JR, Sattar N. Excess Cardiovascular Risk in Type 1 Diabetes Mellitus. Circulation. 2019;139(6):744-7.

2. Schofield J, Ho J, Soran H. Cardiovascular Risk in Type 1 Diabetes Mellitus. Diabetes Ther. 2019;10(3):773-89.

3. Davies MJ, D'Alessio DA, Fradkin J, Kernan WN, Mathieu C, Mingrone G, Rossing P, Tsapas A, Wexler DJ, Buse JB. Management of hyperglycaemia in type 2 diabetes, 2018. A consensus report by the American Diabetes Association (ADA) and the European Association for the Study of Diabetes (EASD). Diabetologia. 2018;61(12):2461-98.

4. Renard CB, Kramer F, Johansson F, Lamharzi N, Tannock LR, von Herrath MG, Chait A, Bornfeldt KE. Diabetes and diabetes-associated lipid abnormalities have distinct effects on initiation and progression of atherosclerotic lesions. J Clin Invest. 2004;114(5):659-68.

5. Nathan DM, Cleary PA, Backlund JY, Genuth SM, Lachin JM, Orchard TJ, Raskin P, Zinman B. Intensive diabetes treatment and cardiovascular disease in patients with type 1 diabetes. $\mathrm{N}$ Engl $\mathrm{J}$ Med. 2005;353(25):2643-53.

6. Teleb M, Popp Switzer M, Elhanafi S, Elfar A, San Juan ZT. Glycemic Control and Excess Cardiovascular Mortality in Type 1 Diabetes. Curr Cardiol Rep. 2016;18(3):29.

7. Ray KK, Seshasai SR, Wijesuriya S, Sivakumaran R, Nethercott S, Preiss D, Erqou S, Sattar N. Effect of intensive control of glucose on cardiovascular outcomes and death in patients with diabetes mellitus: a meta-analysis of randomised controlled trials. Lancet. 2009;373(9677):1765-72.

8. Bebu I, Braffett BH, Pop-Busui R, Orchard TJ, Nathan DM, Lachin JM. The relationship of blood glucose with cardiovascular disease is mediated over time by traditional risk factors in type 1 diabetes: the DCCT/EDIC study. Diabetologia. 2017;60(10):2084-91.

9. Hero C, Svensson AM, Gidlund P, Gudbjornsdottir S, Eliasson B, Eeg-Olofsson K. LDL cholesterol is not a good marker of cardiovascular risk in Type 1 diabetes. Diabet Med. 2016;33(3):316-23.

10. Soedamah-Muthu SS, Chaturvedi N, Toeller M, Ferriss B, Reboldi P, Michel G, Manes C, Fuller JH. Risk factors for coronary heart disease in type 1 diabetic patients in Europe: the EURODIAB Prospective Complications Study. Diabetes Care. 2004;27(2):530-7.

11. Amor AJ, Vinagre I, Valverde M, Urquizu X, Meler E, Lopez E, Alonso N, Pane A, Gimenez M, Codina L, et al. Nuclear magnetic resonance-based metabolomic analysis in the assessment of preclinical atherosclerosis in type 1 diabetes and preeclampsia. Diabetes Res Clin Pract. 2020;171:108548.

12. Verges B. Lipid disorders in type 1 diabetes. Diabetes Metab. 2009;35(5):353-60.

13. James RW, Pometta D. Differences in lipoprotein subfraction composition and distribution between type I diabetic men and control subjects. Diabetes. 1990;39(10):1158-64.

14. Ganjali S, Dallinga-Thie GM, Simental-Mendia LE, Banach M, Pirro M, Sahebkar A. HDL functionality in type 1 diabetes. Atherosclerosis. 2017;267:99-109.

15. Caixas A, Ordonez-Llanos J, de Leiva A, Payes A, Homs R, Perez A. Optimization of glycemic control by insulin therapy decreases the proportion of small dense LDL particles in diabetic patients. Diabetes. 1997;46(7):1207-13. 
16. Otocka-Kmiecik A, Mikhailidis DP, Nicholls SJ, Davidson M, Rysz J, Banach M. Dysfunctional HDL: a novel important diagnostic and therapeutic target in cardiovascular disease? Prog Lipid Res. 2012;51(4):314-24.

17. Schofield JD, France M, Ammori B, Liu Y, Soran H. High-density lipoprotein cholesterol raising: does it matter? Curr Opin Cardiol. 2013;28(4):464-74.

18. Brugnara L, Mallol R, Ribalta J, Vinaixa M, Murillo S, Casserras T, Guardiola M, Vallve JC, Kalko SG, Correig X, et al. Improving Assessment of Lipoprotein Profile in Type 1 Diabetes by $1 \mathrm{H}$ NMR Spectroscopy. PLoS One. 2015;10(8):e0136348.

19. Levey AS, Stevens LA, Schmid CH, Zhang YL, Castro AF 3rd, Feldman HI, Kusek JW, Eggers P, Van Lente $F$, Greene $T$, et al. A new equation to estimate glomerular filtration rate. Ann Intern Med. 2009;150(9):604-12.

20. Mallol R, Amigo N, Rodriguez MA, Heras M, Vinaixa M, Plana N, Rock E, Ribalta J, Yanes O, Masana L, et al. Liposcale: a novel advanced lipoprotein test based on 2D diffusion-ordered 1H NMR spectroscopy. J Lipid Res. 2015;56(3):737-46.

21. Mallol R, Rodriguez MA, Heras M, Vinaixa M, Plana N, Masana L, Morris GA, Correig X. Particle size measurement of lipoprotein fractions using diffusion-ordered NMR spectroscopy. Anal Bioanal Chem. 2012;402(7):2407-15.

22. Llaurado G, Amigo N, Cano A, Ballesta S, Albert L, Mazarico I, Fernandez-Veledo S, Pedro-Botet J, Vendrell J, Gonzalez-Clemente JM. Specific Nuclear Magnetic Resonance Lipoprotein Subclass Profiles and Central Arterial Stiffness in Type 1 Diabetes Mellitus: A Case Control Study. J Clin Med 2019, 8(11).

23. Taskinen MR. Quantitative and qualitative lipoprotein abnormalities in diabetes mellitus. Diabetes. 1992;41(Suppl 2):12-7.

24. Ostlund RE Jr, Semenkovich CF, Schechtman KB. Quantitative relationship between plasma lipids and glycohemoglobin in type I patients. Longitudinal study of 212 patients. Diabetes Care. $1989 ; 12(5): 332-6$.

25. Lipid. and lipoprotein levels in patients with IDDM diabetes control and complication. Trial experience. The DCCT Research Group. Diabetes Care. 1992;15(7):886-94.

26. Perez A, Wagner AM, Carreras G, Gimenez G, Sanchez-Quesada JL, Rigla M, Gomez-Gerique JA, Pou JM, de Leiva A. Prevalence and phenotypic distribution of dyslipidemia in type 1 diabetes mellitus: effect of glycemic control. Arch Intern Med. 2000;160(18):2756-62.

27. Amor AJ, Castelblanco E, Hernandez M, Gimenez M, Granado-Casas M, Blanco J, Soldevila B, Esmatjes E, Conget I, Alonso N, et al. Advanced lipoprotein profile disturbances in type 1 diabetes mellitus: a focus on LDL particles. Cardiovasc Diabetol. 2020;19(1):126.

28. Balling M, Afzal S, Varbo A, Langsted A, Davey Smith G, Nordestgaard BG. VLDL Cholesterol Accounts for One-Half of the Risk of Myocardial Infarction Associated With apoB-Containing Lipoproteins. J Am Coll Cardiol. 2020;76(23):2725-35. 
29. Talayero BG, Sacks FM. The role of triglycerides in atherosclerosis. Curr Cardiol Rep. 2011;13(6):544-52.

30. Colhoun HM, Otvos JD, Rubens MB, Taskinen MR, Underwood SR, Fuller JH. Lipoprotein subclasses and particle sizes and their relationship with coronary artery calcification in men and women with and without type 1 diabetes. Diabetes. 2002;51(6):1949-56.

31. Nordestgaard BG, Tybjaerg-Hansen A. IDL, VLDL, chylomicrons and atherosclerosis. Eur J Epidemiol. 1992;8(Suppl 1):92-8.

32. Shoji T, Nishizawa Y, Kawagishi T, Kawasaki K, Taniwaki H, Tabata T, Inoue T, Morii H. Intermediatedensity lipoprotein as an independent risk factor for aortic atherosclerosis in hemodialysis patients. J Am Soc Nephrol. 1998;9(7):1277-84.

33. Winocour PH, Durrington PN, Bhatnagar D, Ishola M, Mackness M, Arrol S. Influence of early diabetic nephropathy on very low density lipoprotein (VLDL), intermediate density lipoprotein (IDL), and low density lipoprotein (LDL) composition. Atherosclerosis. 1991;89(1):49-57.

34. Bonneau G, Castillo-Rascón MS, Sánchez RA, Pedrozo WR, Castro-Olivera C. IDL cholesterol and lipidic parameters in diabetic patients type 2. Revista Argentina de Endocrinologia y Metabolismo. 2007;44(4):215-22.

35. Verges B. Dyslipidemia in Type 1 Diabetes: AMaskedDanger. Trends Endocrinol Metab. 2020;31(6):422-34.

36. Brunzell JD, Zambon A, Deeb SS. The effect of hepatic lipase on coronary artery disease in humans is influenced by the underlying lipoprotein phenotype. Biochim Biophys Acta. 2012;1821(3):365-72.

37. Emmison N, Zammit VA, Agius L. Triacylglycerol accumulation and secretion in hepatocyte cultures. Effects of insulin, albumin and Triton WR 1339. Biochem J. 1992;285(Pt 2):655-60.

38. Yoon TH, Yamada N, Ishibashi S, Shimano H, Gotoda T, Harada K, Akanuma Y, Murase T, Takaku F. The release of hepatic triglyceride lipase from rat monolayered hepatocytes in primary culture. Endocrinol Jpn. 1990;37(3):437-42.

39. Nakai T, Yamada S, Tamai T, Kobayashi T, Hayashi T, Takeda R. The effects of streptozotocin diabetes on hepatic triglyceride lipase activity in the rat. Metabolism. 1979;28(1):30-40.

40. Elkeles RS, Hambley J. The effects of fasting and streptozotocin diabetes on hepatic triglyceride lipase activity in the rat. Diabetes. 1977;26(1):58-60.

\section{Tables}

Table 1 Clinical and laboratory characteristics of the study subjects 


\begin{tabular}{|c|c|c|c|c|}
\hline Parameters & $\begin{array}{l}\text { Baseline } \\
\text { T1D }\end{array}$ & $\begin{array}{l}\text { Follow-up } \\
\text { T1D }\end{array}$ & $\begin{array}{l}\text { Standardized } \\
\text { difference } b\end{array}$ & $\underset{a}{p \text {-value }}$ \\
\hline \multicolumn{5}{|l|}{$\begin{array}{l}\text { Clinical and biochemical } \\
\text { parameters }\end{array}$} \\
\hline Systolic blood pressure [mm Hg] & $\begin{array}{l}105.9 \\
(15.5)\end{array}$ & $111.0(15.1)$ & -0.34 & 0.416 \\
\hline $\begin{array}{l}\text { Diastolic blood pressure [mm } \\
\mathrm{Hg}]\end{array}$ & $64.1(12.1)$ & $65.4(9.52)$ & -0.12 & 0.765 \\
\hline Waist circumference [cm] & $76.2(6.6)$ & $76.2(8.3)$ & -0.07 & 0.765 \\
\hline Body mass index $\left[\mathrm{kg} / \mathrm{m}^{2}\right]$ & $20.3(1.7)$ & $21.4(1.5)$ & -0.69 & 0.004 \\
\hline Glucose $[\mathrm{mg} / \mathrm{dL}]$ * & $\begin{array}{l}212.9 \\
(56.6)\end{array}$ & $98.7(21.2)$ & 2.29 & \\
\hline $\mathrm{HbA} 1 \mathrm{c}[\%]$ * & $11.5(1.5)$ & $6.2(0.5)$ & 5.03 & \\
\hline Creatinine [mg/dL] & $0.7(0.1)$ & $0.8(0.2)$ & -0.38 & 0.164 \\
\hline GFR-CKD-EPI & $126.7(6.7)$ & $121.6(12.2)$ & 0.48 & 0.198 \\
\hline ALT [U/L] & $18.7(13.2)$ & $13.3(3.8)$ & 0.51 & 0.258 \\
\hline GGT [U/L] & $14.1(3.2)$ & $13.1(3.1)$ & 0.34 & 0.198 \\
\hline Fatty liver index & $7.3(5.1)$ & $7.0(5.5)$ & 0.09 & 0.765 \\
\hline \multicolumn{5}{|l|}{ Lipids and apolipoproteins } \\
\hline Total triglycerides [mg/dL] & $89.9(50.7)$ & $68.7(43.5)$ & 0.45 & 0.272 \\
\hline Total cholesterol [mg/dL] & $\begin{array}{l}174.7 \\
(25.8)\end{array}$ & $153.8(22.7)$ & 0.85 & 0.054 \\
\hline LDL-C [mg/dL] & $\begin{array}{l}106.9 \\
(15.2)\end{array}$ & $89.7(12.8)$ & 1.22 & 0.011 \\
\hline $\mathrm{HDL}-\mathrm{C}[\mathrm{mg} / \mathrm{dL}]$ & $62.7(12.9)$ & $64.7(9.6)$ & -0.15 & 0.563 \\
\hline \multicolumn{5}{|l|}{ Predictive ratios of CVD risk } \\
\hline Total cholesterol/HDL-C & $2.9(0.6)$ & $2.4(0.5)$ & 0.81 & 0.045 \\
\hline LDL-C/HDL-C & $1.8(0.5)$ & $1.4(0.3)$ & 0.85 & 0.012 \\
\hline VLDL-C + LDL-C/HDL-C & $1.9(0.5)$ & $1.5(0.3)$ & 0.83 & 0.011 \\
\hline Triglyceride/HDL-C & $1.5(0.7)$ & $1.1(0.7)$ & 0.54 & 0.164 \\
\hline
\end{tabular}

Data are presented as mean and standard deviation of 12 pairs of samples. 
* pre-established parameters according to inclusion criteria described in the Materials and Methods section.

a Overall p-value by Sign test: 0.0161 , and post hoc test using paired t-test corrected for Benjamini \& Hochberg (false discovery rate). ${ }^{b}$ The standardized mean difference is defined as the difference in mean outcome between groups divided by the standard deviation of outcome; it compares the difference in the mean in units of the standard deviation of both groups. ALT, alanine aminotransferase; CVD, cardiovascular disease; GFR-CKD-EPI, glomerular filtration rate; GGT, gamma-glutamyl transferase; HbA1c, glycated hemoglobin; HDL, high-density lipoprotein; LDL, low-density lipoprotein; VLDL, very-lowdensity lipoprotein.

The standardized mean difference compares the difference in the mean in units of the standard deviation of both groups.

Table 2 Changes in lipid content of serum lipoproteins after optimization of glycemic control

\begin{tabular}{|c|c|c|c|c|c|}
\hline $\begin{array}{l}\text { Lipoprotein } \\
\text { classes }\end{array}$ & Parameters & $\begin{array}{l}\text { Onset } \\
\text { T1D }\end{array}$ & $\begin{array}{l}\text { Follow-up } \\
\text { T1D }\end{array}$ & $\begin{array}{l}\text { Standardized } \\
\text { difference } b\end{array}$ & $\underset{a}{p \text {-value }}$ \\
\hline \multirow[t]{2}{*}{ VLDL } & $\begin{array}{l}\text { Cholesterol } \\
\text { [mM] }\end{array}$ & $\begin{array}{l}0.22 \\
(0.12)\end{array}$ & $0.17(0.12)$ & 0.43 & 0.045 \\
\hline & $\begin{array}{l}\text { Triglycerides } \\
\text { [mM] }\end{array}$ & $\begin{array}{l}0.46 \\
(0.18)\end{array}$ & $0.36(0.17)$ & 0.56 & 0.021 \\
\hline \multirow[t]{2}{*}{ IDL } & $\begin{array}{l}\text { Cholesterol } \\
\text { [mM] }\end{array}$ & $\begin{array}{l}0.22 \\
(0.09)\end{array}$ & $0.12(0.06)$ & 1.22 & $<0.001$ \\
\hline & $\begin{array}{l}\text { Triglycerides } \\
\text { [mM] }\end{array}$ & $\begin{array}{l}0.10 \\
(0.03)\end{array}$ & $0.07(0.03)$ & 0.99 & $<0.001$ \\
\hline \multirow[t]{2}{*}{ LDL } & $\begin{array}{l}\text { Cholesterol } \\
\text { [mM] }\end{array}$ & $\begin{array}{l}2.76 \\
(0.39)\end{array}$ & $2.32(0.33)$ & 1.22 & 0.004 \\
\hline & $\begin{array}{l}\text { Triglycerides } \\
\text { [mM] }\end{array}$ & $\begin{array}{l}0.14 \\
(0.03)\end{array}$ & $0.09(0.03)$ & 1.59 & $<0.001$ \\
\hline \multirow[t]{2}{*}{ HDL } & $\begin{array}{l}\text { Cholesterol } \\
\text { [mM] }\end{array}$ & $\begin{array}{l}1.62 \\
(0.37)\end{array}$ & $1.67(0.25)$ & -0.15 & 0.463 \\
\hline & $\begin{array}{l}\text { Triglycerides } \\
\text { [mM] }\end{array}$ & $\begin{array}{l}0.17 \\
(0.08)\end{array}$ & $0.14(0.05)$ & 0.27 & 0.045 \\
\hline
\end{tabular}

Data are presented as mean and standard deviation of 12 pairs of samples. ${ }^{a}$ Overall p-value by Sign test: $<0.001$ and post hoc test using paired t-test corrected for Benjamini \& Hochberg (false discovery rate). ${ }^{b}$ The standardized mean difference is defined as the difference in mean outcome between groups divided by the standard deviation of outcome; it compares the difference in the mean in units of the standard 
deviation of both groups. HDL, high-density lipoprotein; IDL, intermediate-density lipoprotein; LDL, lowdensity lipoprotein; VLDL, very-low-density lipoprotein.

Table $\mathbf{3}$ Changes in size distribution and particle diameter in each lipoprotein class after optimization of glycemic control

\begin{tabular}{|c|c|c|c|c|c|}
\hline $\begin{array}{l}\text { Lipoprotein } \\
\text { classes }\end{array}$ & Parameters & Onset T1D & $\begin{array}{l}\text { Follow-up } \\
\text { T1D }\end{array}$ & $\begin{array}{l}\text { Standardized } \\
\text { difference }^{b}\end{array}$ & $\underset{a}{p \text {-value }}$ \\
\hline \multirow[t]{5}{*}{ VLDL } & Total [nM] & $28.83(11.7)$ & $23.6(10.5)$ & 0.46 & 0.085 \\
\hline & Large [nM] & $0.77(0.24)$ & $0.64(0.26)$ & 0.52 & 0.122 \\
\hline & Medium [nM] & $3.47(1.78)$ & $2.10(1.48)$ & 0.82 & 0.033 \\
\hline & Small [nM] & $24.60(10.0)$ & $20.89(8.91)$ & 0.39 & 0.133 \\
\hline & $\begin{array}{l}\text { Particle size } \\
\text { [nm] }\end{array}$ & $42.30(0.29)$ & $42.0(0.23)$ & 1.05 & 0.122 \\
\hline \multirow[t]{5}{*}{ LDL } & Total [nM] & $\begin{array}{l}745.83 \\
(103.64)\end{array}$ & $\begin{array}{l}625.93 \\
(89.76)\end{array}$ & 1.23 & 0.007 \\
\hline & Large [nM] & $\begin{array}{l}122.77 \\
(16.33)\end{array}$ & $\begin{array}{l}106.90 \\
(11.51)\end{array}$ & 1.11 & 0.036 \\
\hline & Medium [nM] & $\begin{array}{l}237.19 \\
(46.85)\end{array}$ & $\begin{array}{l}157.41 \\
(60.63)\end{array}$ & 1.46 & 0.007 \\
\hline & Small [nM] & $\begin{array}{l}385.86 \\
(51.76)\end{array}$ & $\begin{array}{l}361.62 \\
(48.62)\end{array}$ & 0.49 & 0.273 \\
\hline & $\begin{array}{l}\text { Particle size } \\
\text { [nm] }\end{array}$ & $21.22(0.17)$ & $21.02(0.29)$ & 0.86 & 0.138 \\
\hline \multirow[t]{5}{*}{ HDL } & Total [mM] & $30.26(7.92)$ & $30.37(4.88)$ & -0.01 & 0.936 \\
\hline & Large [mM] & $0.31(0.06)$ & $0.29(0.05)$ & 0.24 & 0.667 \\
\hline & Medium [mM] & $11.8(2.54)$ & $11.71(2.31)$ & 0.03 & 0.936 \\
\hline & Small [mM] & $18.2(5.53)$ & $18.37(3.60)$ & -0.04 & 0.936 \\
\hline & $\begin{array}{l}\text { Particle size } \\
\text { [nm] }\end{array}$ & $8.32(0.05)$ & $8.31(0.08)$ & 0.18 & 0.829 \\
\hline
\end{tabular}

Data are presented as mean and standard deviation of 12 pairs of samples.

a Overall $p$-value by Sign test: $<0.001$ and post hoc test using paired t-test corrected for Benjamini \& Hochberg (false discovery rate). ${ }^{b}$ The standardized mean difference is defined as the difference in mean outcome between groups divided by the standard deviation of outcome; it compares the difference in the 
mean in units of the standard deviation of both groups. HDL, high-density lipoprotein; IDL, intermediatedensity lipoprotein; LDL, low-density lipoprotein; VLDL, very-low-density lipoprotein.

\section{Supplementary Files}

This is a list of supplementary files associated with this preprint. Click to download.

- Additionalfile1.docx

- Additionalfile2.docx

- Additionalfile3.docx

- Additionalfile4.docx

- Additionalfile5.docx

- AdditionalFile6.tif

- AdditionalFile7.tif 